\title{
Low-burden biological feedback controllers for near-perfect adaptation
}

\author{
Harrison Steel and Antonis Papachristodoulou *
}




\begin{abstract}
The robustness and reliability of synthetic biological systems can be substantially improved by the introduction of feedback control architectures that parallel those employed in traditional engineering disciplines. One common control goal is adaptation (or disturbance rejection), which refers to a system's ability to maintain a constant output despite variation in some of its constituent processes (as frequently occurs in noisy cellular environments) or external perturbations. In this paper we propose and analyse a control architecture that employs Integrase and Excisionase proteins to invert regions of DNA, and an mRNA-mRNA annihilation reaction. Combined, these components approximate the functionality of a switching controller (as employed in classical control engineering) with three distinct operational modes. We demonstrate that this system is capable of near-perfect adaptation to variation in rates of both transcription and translation, and can also operate without excessive consumption of cellular resources. The system's steady state behaviour is analysed, and limits on its operating range are derived. Deterministic simulations of its dynamics are presented, and are then extended to the stochastic case which treats biochemical reactions as discrete events.
\end{abstract}

Keywords: synthetic biology, biological control, adaptation, switching controller, recombinase enzymes 
Recent work in synthetic biological research has focused on the development of control architectures that parallel those employed in other engineering disciplines $(1,2)$, with the aim of improving the robustness and reliability of engineered biological systems (3). A central goal of this work has been the creation of biological systems capable of adaptation (e.g. to achieve disturbance rejection). This can be loosely defined as the ability of a system to maintain one variable's value (for example, the concentration of a biochemical species) constant as the parameters or behaviour of a system with which it interacts varies. Adaptation has been observed to occur in some natural systems $(4,5)$, structural design constraints that must be satisfied to achieve this functionality have been outlined $(6,7)$, and control architectures that achieve near-perfect adaptation have recently been experimentally demonstrated (8). Similar feedback circuits have also been created using a range of biological components, such as synthetic protein scaffolds (9) and sigma/anti-sigma factors $(8,10)$. However, at present these implementations struggle to overcome challenges such as leakiness of their "integrator" components (which are required for perfect adaptation) due to dilution/degradation of their constitutent components $(11,12)$. This has led to the proposition of alternative approaches that, though not allowing perfect adaptation, nevertheless come close (for example, by employing systems that exhibit an ultrasensitive input-output mapping (13)).

In this paper we propose and analyse a different control system which provides a range of benefits when compared to previously proposed architectures: It allows near-perfect rejection of disturbances to both cellwide translation rates (frequently arising due to ribosome sequestration $(14,15))$ and transcription rate (arising due to fluctuations in plasmid copy-number (16)). Furthermore, our system is designed to operate in a "slack region" where (at equilibrium) the expression rate of its constituent proteins is minimised. This reduces the burden (consumption of cellular resources) that it imparts upon its host cell, which could otherwise adversely impact cellular growth and the behaviour of other synthetic circuits $(17,18,19,20)$.

The system proposed achieves these goals by using two biological motifs employed in recent experimental work: First, Integrase/Excisionase proteins (which have been used to implement information storage (21, 22 ), logic (23), and feedback control (24)) are employed to flip a DNA "register" flanked by recognition sites. This aspect of our design is motivated by a desire to side-step the impact of dilution effects experienced by controllers for which internal states are stored as protein concentrations; DNA registers avoid this as their state is maintained during duplication. Second, an mRNA-mRNA annihilation reaction provides functionality similar to a "subtraction" junction from traditional control engineering. Biological feedback control structures which employ similar mRNA-sRNA annihilation interactions have been theoretically $(11,25)$ and experimentally $(26,27,28)$ demonstrated in the literature. The novelty of the present work thus focuses on the interconnection of these two biological motifs, resulting in a controller that benefits from the useful properties of each motif. The architecture we develop is inspired by switching control schemes (commonly employed in classical control engineering $(29,30)$ ), which move between discrete operational modes depending on a reference signal.

\section{Results}

\section{System Description}

In this study we propose a biological implementation of a switching controller (Fig. 1a) which aims to regulate its output $G$ to adapt to cell-wide disturbances $\Delta$ in translation rate (which could arise due to varying ribosome availability) and $\Theta$ in transcription rate of plasmid encoded genes (which could arise due to varying plasmid copy number). Fig. 1b presents a block diagram for the system, outlining the interactions between components as well as the points at which these disturbances are introduced. Disturbance rejection is achieved by regulating the number of plasmid DNA registers in their $\mathrm{ON}$ position (which determines the transcription rate of the controller's output, $G$ ) via switching the controller between three operating modes (Fig. 1e).

Our proposed system is comprised of a plasmidencoded component (which includes a flippable DNA register and genes for the system output $G$ and mRNA $z$ ), and a genome encoded component (which includes mRNA $\left.z^{*}\right)$. Also encoded in the genome is a measurement species $D$, which is responsible for coupling variations in translation rate $(\Delta)$ to the expression of $z$. Control functionality is implemented using Integrase $(I)$ and Excisionase $(X)$ proteins that flip a DNA register flanked by recognition sites (Fig. 1c), regulating the proportion of DNA registers that are in their ON position ( $p$, which we initially treat as a continuous variable). $I$ alone can flip DNA from ON to OFF, whilst both $I$ and $X$ are required for the reverse flipping operation (24). From registers in their ON position the output $G$ is transcribed, as is mRNA $z$ (encoding $I$ ). Meanwhile, an mRNA $z^{*}$ that encodes both $I$ and $Z$ is transcribed continuously from the genome, as is measurement species $D$. The mRNAs $z$ and $z^{*}$ irreversibly bind to each other in such a way that translation from each is supressed.

The overall design can be conceptualised as an approximation of a switching controller $(29,30)$ which operates in three distinct modes, $m_{0}, m_{1}$, and $m_{2}$ (Fig. 1e) defined as follows: When production rates of $z$ and $z^{*}$ are balanced little $I$ or $X$ is produced, meaning flipping is slow (both $R_{o n}$ and $R_{\text {off }}$ are small) and $p$ remains approximately constant (mode $m_{0}$ ). However, 

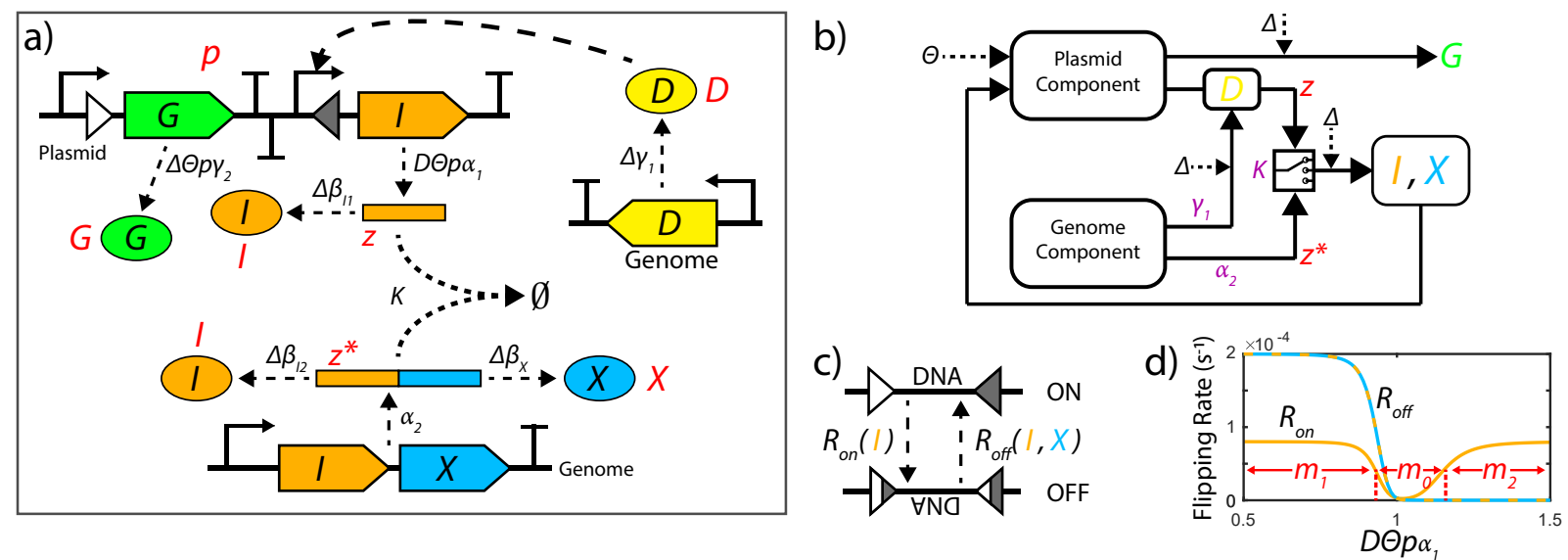

e)

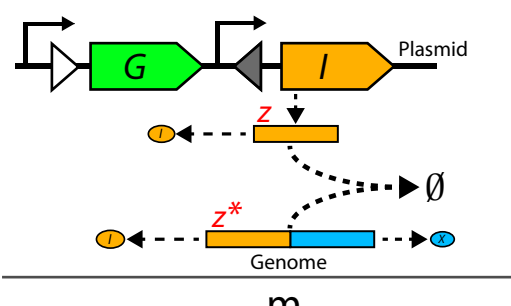

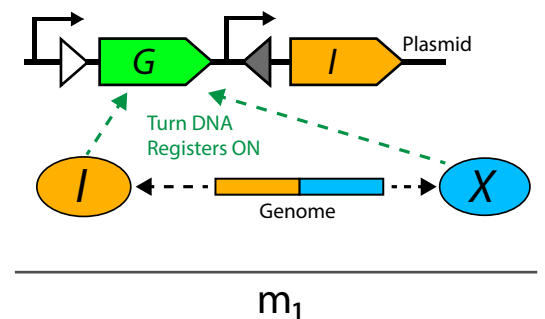

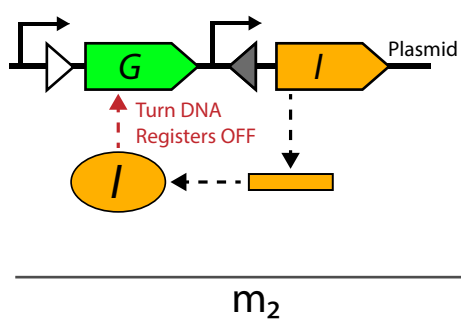

Figure 1: Proposed Control Architecture a) The Integrase/Excisionase control architecture proposed in this work, which is designed to reject disturbances to transcription $(\Theta)$ and translation $(\Delta)$ rates. It is comprised of interacting mRNA species $\left(z, z^{*}\right)$ which mutually annihilate, an output protein $(G)$, a measurement protein ( $D$, which couples translation variation to the transcription of $z$ ), and recombinase enzymes $(I, X)$ which flip DNA registers (flanked by converging triangles). State variables from Eq (1) are in red, other parameters are as described in Table 1. b) Block diagram of the control system, including a selector junction for the system's three modes. Important parameters for tuning are highlighted in purple. c) Illustration of Integrase/Excisionase mediated flipping of a DNA register between recognition sites (triangles). Solid triangles represent initial DNA recognition sites (requiring $I$ for flipping), half-coloured triangles represent flipped DNA sites (requiring $I$ and $X$ for reverse flipping). d) DNA flipping rates as a function of steady-state $I$ and $X$ values when the production rate of $z\left(D \Theta p \alpha_{1}\right)$ is set to a fixed value, demonstrating a "slack region" $\left(m_{0}\right.$, where $\left.D \Theta p \alpha_{1} \approx 1\right)$ near which both flipping rates are small. e) Illustration of the controller's three switching modes. In mode $m_{0}$ production of mRNA species $z$ and $z^{*}$ is balanced, meaning most mRNA are bound to one another (preventing translation), and thus DNA flipping is slow. When the system is disturbed (by a change in cell-wide translation rate or plasmid copy number) mRNA production is out of balance and the system enters either mode $m_{1}$ (to increase output $G$ ) or $m_{2}$ (to decrease $G$ ). In $m_{1}$ genome-expressed Integrase and Excisionase work together to flip DNA registers to their ON position, whilst in $m_{2}$ plasmid-expressed Integrase flips registers to their OFF position.

the concentration of $z$ (and $G$ ) can be disturbed by a change in either transcription rate via $\Theta$, or global translation rate via $\Delta$ (since the production of $z$ is regulated by the measurement protein species $D$ ). When this inbalance in $z$ and $z^{*}$ arises either $I$ (if $z \gg z^{*}$, mode $m_{2}$ ) or $I$ and $X$ (if $z \ll z^{*}$, mode $m_{1}$ ) will be produced, which leads to flipping of DNA registers (varying $p$ ) to return expression of $z$ and $G$ to its desired setpoint. The boundaries between different control modes are illustrated numerically in Fig. 1d, where we fix the production rate of $z\left(D \Theta p \alpha_{1}\right)$ and then calculate corresponding steady-state $I$ and $X$ concentrations: When $D \Theta p \alpha_{1} \approx 1$ (mode $m_{0}$ ) we have $R_{o n}, R_{\text {off }} \approx 0$ and so minimal flipping occurs in either direction.

\section{ODE Model}

The proposed control system can be described using a system of ordinary differential equations (ODEs) of the form:

$$
\begin{aligned}
\dot{D} & =\Delta \gamma_{1}-\delta_{2} D \\
\dot{z} & =D \Theta p \alpha_{1}-\delta_{1} z-K z z^{*} \\
z^{*} & =\alpha_{2}-\delta_{1} z^{*}-K z z^{*} \\
\dot{I} & =\Delta \beta_{I 1} z+\Delta \beta_{I 2} z^{*}-\delta_{2} I \\
\dot{X} & =\Delta \beta_{X} z^{*}-\delta_{2} X \\
\dot{G} & =\Delta \Theta p \gamma_{2}-\delta_{3} G \\
\dot{p} & =R_{o f f}(I, X)(1-p)-R_{o n}(I)(p)
\end{aligned}
$$

where state variables $D, z, z^{*}, I, X, G$ are concentrations as defined previously and $p \in[0,1]$ is the proportion of registers in their "ON" state. $\alpha$ 's are transcription rates, $\beta$ 's are translation rates, $\gamma$ 's are lumped transcription/translation rates, $\delta$ 's are combined rates 

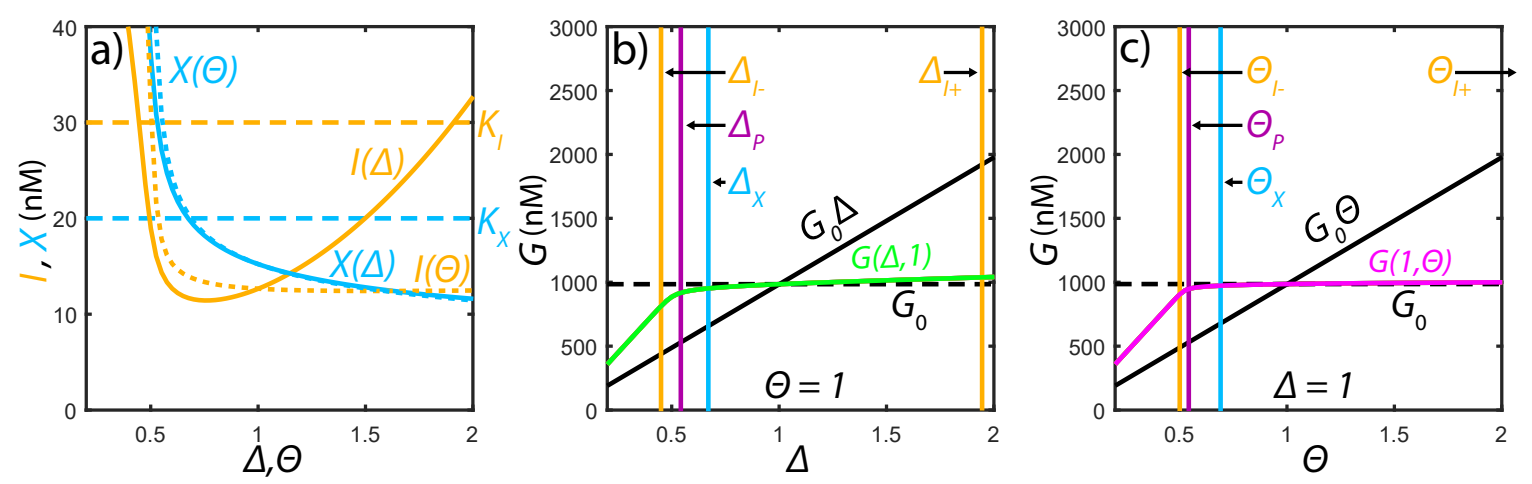

Figure 2: Steady-state Analysis. a) Steady-state $I$ and $X$ concentrations as a function of $\Delta(I(\Delta)$ and $X(\Delta)$, solid lines, $\Theta=1)$ or $\Theta(I(\Theta)$ and $X(\Theta)$, dotted lines, $\Delta=1)$. b) System steady-state output $(G(\Delta, 1))$ as a function of $\Delta$ with $\Theta=1$, compared to its target value $\left(G_{0}\right.$, black dashed line) and the result from an un-controlled case $\left(G_{0} \Delta\right)$. Limiting values of $\Delta$ as described in the text are indicated by vertical lines. Control capability quickly declines for $\Delta$ values below the saturation point $\Delta_{p}$. c) Similar to panel (b), but with $\Delta=1$ and $\Theta$ varying. $\Theta_{I+} \approx 500$ is not pictured.

\begin{tabular}{c|l} 
Parameter & Description \\
\hline$\Theta$ & Transcriptional disturbance magnitude. \\
$\alpha_{1,2}$ & Translational disturbance magnitude. \\
$\beta_{I 1, I 2, X}$ & Transcription rates. \\
$\gamma_{1,2}$ & Lumplation rates. \\
$K$ & rate. \\
$K_{I, X}$ & mRNA-mRNA binding rate. \\
$\delta_{1,2,3}$ & Integrase/Excisionase equilibrium con- \\
& mRNA/protein combined degradation \\
$r_{\text {on }, o f f}$ & Rates of DNA inversion. \\
$z, z^{*}$ & mRNA concentrations. \\
$G, I, X, D$ & Protein concentrations. \\
$p$ & Fraction of plasmids in "ON" position. \\
$\hat{p}$ & Number of plasmids in "ON" position. \\
$p_{T}$ & Total number of plasmids per cell.
\end{tabular}

Table 1: Summary of model parameters and state variables. For numerical values see Table S1. of degradation/dilution, and $K$ is the binding rate between mRNAs. The parameter $\Delta$ is used to introduce disturbances in global (cell-wide) translation rate, and $\Theta$ introduces disturbances in transcription from the plasmid encoding $G$ and $z$. Initially we have $\Delta, \Theta=$ 1 ; variation from this value represents introduction of a disturbance. Integrase/Excisionase mediated DNA flipping is described by:

$$
\begin{aligned}
R_{o f f}(I, X) & =r_{o f f} f_{X}(X) f_{I}(I) \\
R_{o n}(I) & =r_{\text {on }} f_{I}(I) \\
f_{X}(X) & =\frac{X^{4}}{K_{X}^{4}+X^{4}} \\
f_{I}(I) & =\frac{I^{4}}{K_{I}^{4}+I^{4}}
\end{aligned}
$$

where $r_{o n, o f f}$ are scaling factors for the rates of DNA inversion, $K_{I, X}$ are equilibrium constants that determine the concentration of each protein required for the half-maximum flipping rate to be achieved, and the order four Hill functions arise due to four molecules of each protein being required (one dimer at each recognition site) to perform DNA inversion $(24,31)$. A summary of the model parameters is provided in Table 1 , and the numerical values used in simulations are discussed in Supplementary Section 1.

\section{Steady-state Analysis}

We can solve $\mathrm{Eq}(1)$ at steady-state to eliminate variables $D, z^{*}, I$, and $X$, finding that the equilibrium concentration of $z$ must satisfy:

$$
\begin{aligned}
\frac{\alpha_{1} \gamma_{1} \Delta \Theta p}{\delta_{2}} & =\delta_{1} z+K z \mu(z) \\
p & =\frac{f_{X}\left(\Delta \beta_{X} \mu(z) / \delta_{2}\right)}{\frac{r_{o n}}{r_{o f f}}+f_{X}\left(\Delta \beta_{X} \mu(z) / \delta_{2}\right)} \\
\mu(z) & =\frac{\alpha_{2}}{\delta_{1}+K z}
\end{aligned}
$$


where we note that the factor $\Delta \Theta p$ (proportional to the production rate of $G$ ) appears on the left hand side of Eq (3a). If we have $K z \gg \delta_{1}$ (i.e. most mRNA binds to its complementary mRNA prior to degrading) then the right hand side of $\mathrm{Eq}(3 \mathrm{a})$ can be simplified to give $p \Delta \Theta \propto \delta_{1} z+\alpha_{2}$, meaning that $G$ will reject changes in $\Delta, \Theta$ so long as $\delta_{1} z$ does not vary significantly. This can be partially achieved by minimising $\delta_{1}$ (as in other biological circuits targeting adaptation (11)), as well as by minimising the sensitivity of the steady-state value of $z$ to changes in $\Delta$ and $\Theta$, which we analyse in Supplementary Section 2 .

This architecture's ability to reduce variation in the steady-state $z$ value (and hence the term $\delta_{1} z$ ) can be conceptually understood as arising from "ultrasensitive" behaviour due to $z$ entering the first term in Eq (3b) through the fourth-order Hill function $f_{X}$. Other biological feedback controllers have been proposed which utilise ultrasensitivity to provide similar adaptive behaviour (13), though the "ultrasensitive" component is typically implemented using a zerothorder process (such as a balanced phosphorylation cycle) or high-cooperativity transcription factor regulation (32). We define our system's target as being the steady-state value $G_{0}$, determined by Eq (1) when $\Delta, \Theta$ $=1$; our control system aims to make the difference between its output and this target small for disturbances $\Delta$ and $\Theta$, (i.e. the steady-state error $\left|G(\Delta, \Theta)-G_{0}\right|$ ).

In our previous work (24) feedback controllers were built using Integrase/Excisionase proteins in order to reduce the influence of noise sources upon a circuit's output. In (24) the proportion of registers in their active state was (at equilibrium) maintained by continuous flipping between states (i.e. $I$ and $X$ were both large and $R_{o n}$ and $R_{o f f}$ were balanced). In contrast, the controller outlined herein is designed to move between different switching modes (Fig. 1e), which drive its state toward a "slack region" in which Integrase/Excisionase concentrations are small. Thus, at (or near) its equilibrium our controller produces little of the control species $I$ and $X$, minimising its burden upon the cell.

\section{Performance Bounds}

We can bound the controller's slack region (i.e. approximate the boundaries of $m_{0}$ ) by maximal concentrations of $I$ and $X$, which we define as the point at which flipping rates are $50 \%$ of their maximum (i.e. $X=K_{X}$ or $I=K_{I}$ such that $\left.f_{X}=f_{I}=0.5\right)$. In this slack region we have $I<K_{I}$ and $X<K_{X}$ and thus flipping occurs slowly. The system leaves this slack region (into either $m_{2}$ with $I>K_{I}$, or $m_{1}$ with $X>K_{X}$ and $I>K_{I}$ ) for only short periods of time (immediately following a change in $\Delta$ or $\Theta$ ), during which fast convergence (variation in $p$ ) occurs to bring the output $G$ back toward $G_{0}$. To assess the range of $\Delta$ or $\Theta$ values for which the system's steady state lies in this slack region we solve Eq (1c) and either Eq (1d) or (1e) for $z$ at steady state giving

$$
\begin{aligned}
z_{X} & =\frac{\Delta \alpha_{2} \beta_{X}-K_{X} \delta_{1} \delta_{2}}{K_{X} K \delta_{2}} \\
z_{I+, I-} & =\frac{K K_{I} \delta_{2}-\Delta \beta_{I 1} \delta_{1} \pm \sqrt{s}}{2 \Delta K \beta_{I 1}} \\
s & =\left(K K_{I} \delta_{2}+\Delta \beta_{I 1} \delta_{1}\right)^{2}-4 \Delta^{2} \alpha_{2} \beta_{I 1} \beta_{I 2} K
\end{aligned}
$$

and substitute these expressions into Eq (3). We can solve the resulting equation for $\Delta$ (with $\Theta=1$ ) or $\Theta$ (with $\Delta=1$ ) to yield $\Delta_{I-, I+, X}$ and $\Theta_{I-, I+, X}$ corresponding to $z_{I-, I+, X}$ respectively. This gives an acceptable range of disturbances $\max \left[\Delta_{I-}, \Delta_{X}\right]<\Delta<$ $\Delta_{I+}$ (and similar for $\Theta$ ). Thus, when disturbances are in this range (considering variation to $\Delta$ and $\Theta$ separately) the system's equilibrium lies in a region within which both $I<K_{I}$ and $X<K_{X}$ at steady-state. This region is illustrated in Fig. 2a where steady state $I$ and $X$ concentrations are plot for varying disturbances; the various limiting values correspond to intersections between functions (e.g. $\Delta_{I-,+}$ are the $\Delta$ values at which $I$ crosses $K_{I}$ ). However, because we have not considered saturation of $p$ we have as yet made no guarantee that these limits can actually be reached prior to controller saturation.

We can constrain the magnitude of disturbances that the control system can reject without saturating by examining its performance at limiting values of $p$. As $\Delta, \Theta$ increase the value of the product $\Delta \Theta p$ in Eq (1f) can be maintained by setting an appropriately small $p$. However, though the system does not run out of actuating potential in this case (i.e. we never reach $p=0)$, the steady-state error may increase. For the case where $\Delta, \Theta \rightarrow 0$ the value of $\Delta \Theta p$ can only be maintained up to the point where $z=0$ and almost all registers are in their ON position. We can solve Eq (3) to find the steady state value $\hat{z}$ when $\Delta=\Theta=1$, which we then substitute into:

$$
\begin{aligned}
\frac{f_{X}\left(\beta_{X} \hat{\mu} / \delta_{2}\right)}{\frac{r_{o n}}{r_{o f f}}+f_{X}\left(\beta_{X} \hat{\mu} / \delta_{2}\right)} & =\Theta \Delta \frac{f_{X}\left(\Delta \beta_{X} \alpha_{2} / \delta_{1} \delta_{2}\right)}{\frac{r_{o n}}{r_{o f f}}+f_{X}\left(\Delta \beta_{X} \alpha_{2} / \delta_{1} \delta_{2}\right)} \\
\hat{\mu} & =\frac{\alpha_{2}}{\delta_{1}+K \hat{z}}
\end{aligned}
$$

in which the right hand side of Eq (5a) is the saturated signal $\Delta \Theta p$ when $z=0$. Solving $\operatorname{Eq}(5)$ for $\Delta$ (with $\Theta=1$ ) or $\Theta$ (when $\Delta=1$ ) gives minimal values $\Delta_{p}$ and $\Theta_{p}$ respectively that the system could compensate for without saturating, though steady-state error will again increase as this value is approached.

In Fig. 2b,c we analytically calculate the steady state outputs of Eq (1) (denoted $G(\Delta, \Theta)$ ) over a range of $\Delta$ and $\Theta$ values. The various limiting values of $\Delta$ and $\Theta$ described previously are plot as vertical lines, as is the expected output $\left(G_{0} \Delta \Theta\right)$ that would result without the controller's action (i.e. if $G$ expression was independent of $p$ ). We observe that the system is able to reject a disturbance in $\Delta$, and operates in the intended "slack region" for a wide range of $\Delta$. Rejection 

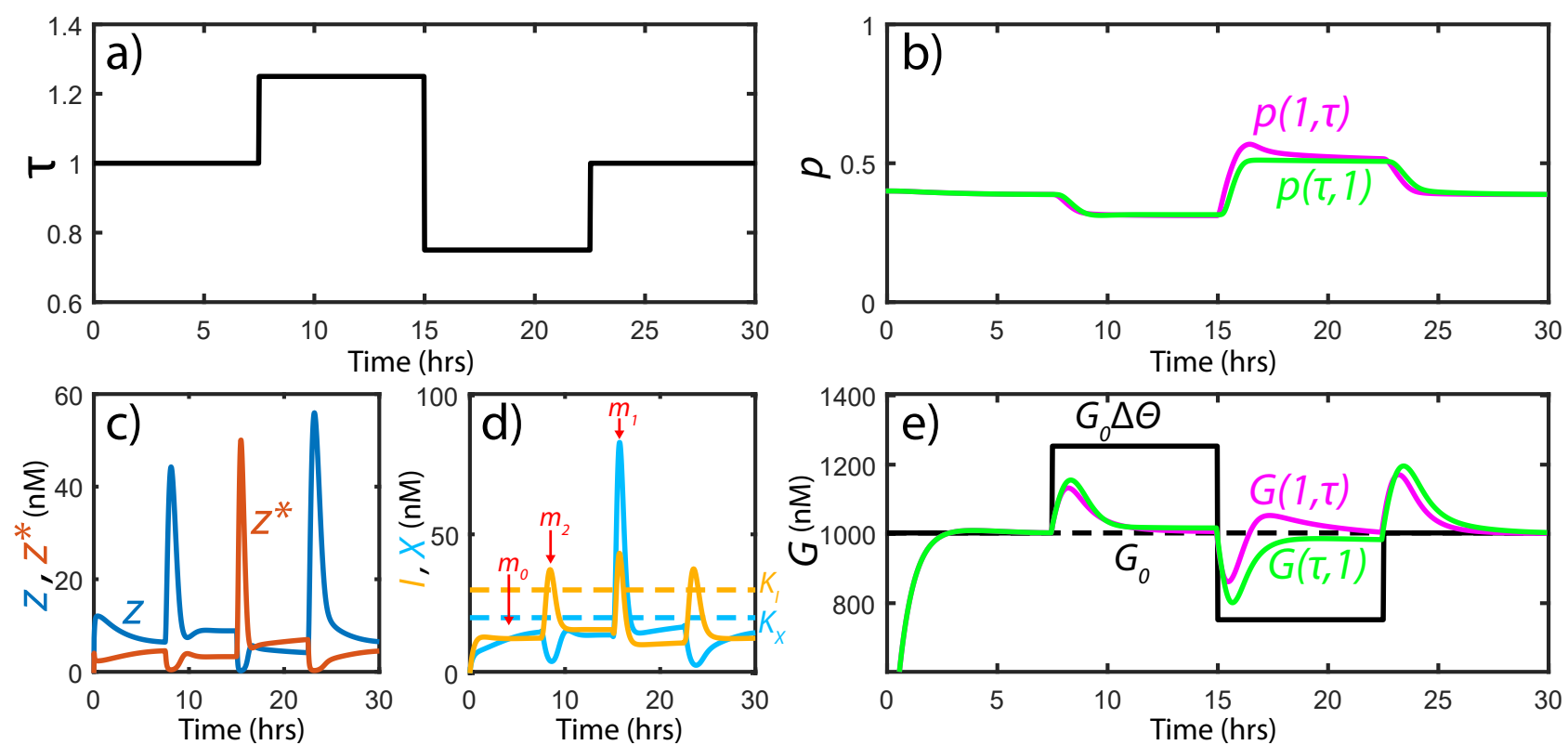

Figure 3: Dynamic simulation of disturbance rejecting behaviour. a) Disturbance profile $\tau$ substituted for $\Delta$ or $\Theta$ in subsequent sub-figures. b) The proportion of plasmids in their ON state $(p)$ varies in response to disturbance in $\Delta(G(\tau, 1))$ or $\Theta(G(1, \tau))$. c) The concentration of each mRNA species and $\mathbf{d})$ concentration of $I$ and $X$ for $\Delta=\tau, \Theta=1$. Spikes in concentration appear immediately following application of a disturbance, which push the production rates of $z$ and $z^{*}$ out of balance. Example points at which the system is in each of its operating modes $m_{0,1,2}$ are labelled. e) System output $G$, demonstrating near-perfect rejection of disturbance to either $\Delta$ or $\Theta$. $G_{0} \Delta \Theta$ indicates the system's expected steady-state output if no control system was in place.

of a disturbance to $\Theta$ is even better (i.e. the slope of $G(1, \Theta)$ is closer to 1$)$, and the slack region extends to much higher values of $\Theta$ (the reason for this is outlined in Supplementary Section 2).

\section{Closed Loop Stability}

We can investigate the stability of the system in Eq (1) by assuming that at equilibrium (which lies in mode $m_{0}$ ) each individual term in the equation for $\dot{p}$ is zero (i.e. $R_{o f f}, R_{o n} \approx 0$ ). Linearising about this point (in terms of $\left.D, z, z^{*}, I, X\right)$ gives the Jacobian:

$\mathbf{J}=\left[\begin{array}{ccccc}-\delta_{2} & 0 & 0 & 0 & 0 \\ -\Theta p \alpha_{1} & -\delta_{1}-K z^{*} & -K z & 0 & 0 \\ 0 & -K z^{*} & -\delta_{1}-K z & 0 & 0 \\ 0 & \Delta \beta_{I 1} & \Delta \beta_{I 2} & -\delta_{2} & 0 \\ 0 & 0 & \Delta \beta_{X} & 0 & -\delta_{2}\end{array}\right]$

which has eigenvalues $\lambda_{1,2,3}=-\delta_{2}$ and

$$
\lambda_{4,5}=-\delta_{1},-\delta_{1}-K\left(z+z^{*}\right)
$$

and thus all system poles (eigenvalues of Eq (6)) are in the left half plane, meaning this equilibrium is stable. We can numerically evaluate the eigenvalues for the complete system's Jacobian (i.e. including $p$ in Eq (6)) about its equilibrium when $\Delta=\Theta=1$, which returns (approximately) the same eigenvalues as above, and an additional sixth value of $\lambda_{6}=-4.4 \times 10^{-6}$ (corresponding to the slower, stable, timescale), which is as expected much smaller than $\delta_{2}$ (previously the eigenvalue of smallest magnitude).

\section{Dynamic Simulation}

In Fig. 3 we numerically integrate the system in Eq (1), applying a time-varying disturbance profile $\tau(t)$ (Fig. 3a) to $\Delta$ (with $\Theta=1, G(\tau, 1)$ ) and then to $\Theta$ (with $\Delta=1, G(1, \tau)$ ). Following a step change in $\tau$ the system rapidly varies $p$ (Fig. $3 \mathrm{~b}$ ), which is driven by fluctuating mRNA concentrations (Fig. 3c) which govern expression of $I$ and $X$ (Fig. 3d). We observe that the system is achieving the intended switching behaviour: $I$ and $X$ concentrations spike for short periods of time, rapidly driving $p$ close to its new equilibrium, and then remain below $K_{I}$ and $K_{X}$ respectively. The system output (Fig. 3e) converges close to its pre-adaptation level following each disturbance, though this occurs over a longer time-scale since protein $G$ is assumed to be stable ( $\delta_{3}$ is small).

\section{Stochastic Simulation}

Though in previous sections we have assumed that $p$ is a continuous variable, in practice a finite number of DNA registers will be present within a given cell (e.g. one, or perhaps a couple, per plasmid). We investigate the impact of discretising $p$ by setting:

$$
\hat{p}=\operatorname{round}\left(p \times p_{T}\right)
$$

and then substituting $p=\hat{p} / p_{T}$ in Eq (1). Here $p_{T}$ is the copy number (per cell) of the flippable DNA register, and the round function returns its argument rounded to the nearest integer. We solve Eq (1) with this modification to find the steady-state value of $G$ 

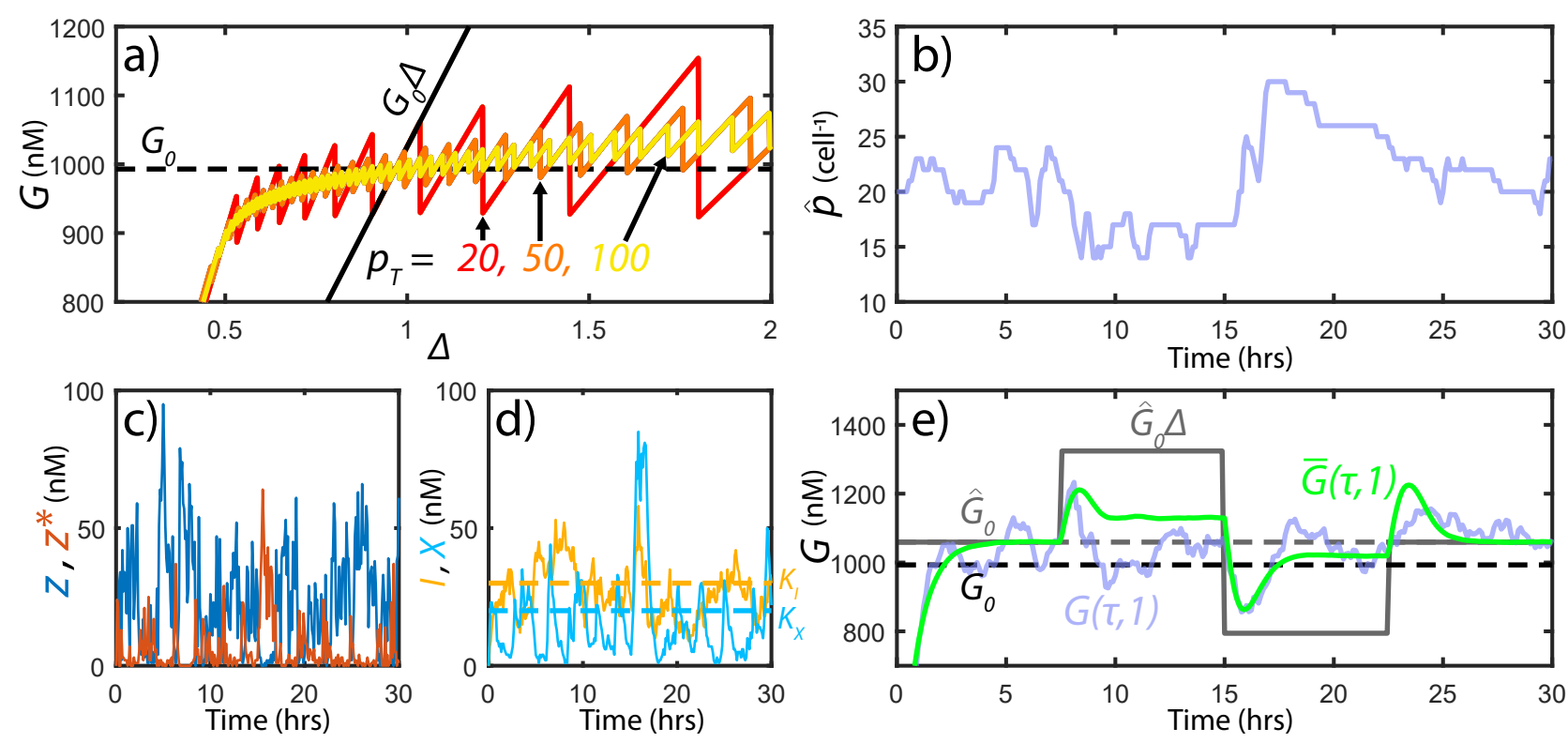

Figure 4: Stochastic simulation of disturbance rejecting behaviour. a) The dependence of our system's behaviour on the copy number of DNA registers $\left(p_{T}\right)$ simulated as described in the text. Other quantities plot parallel those in Fig. 2b. b-d) Similar to Fig. 3b-d but for a single stochastic simulation run and with $\Theta=1$. In panel b the total number of ON registers $(\hat{p})$ is plot, rather than the ON proportion of total registers $(p)$. e) System output $G$ for a single stochastic simulation $(G(\tau, 1))$ and the average output of 3000 stochastic simulations $(\bar{G}(\tau, 1))$. The target output (mean steady-state $G$ value when $\Delta=1$ ) for the stochastic case $\left(\hat{G}_{0}\right)$ differs to that for the deterministic case $\left(G_{0}\right.$, see Fig. 3e) as described in the text.

as $\Delta$ is varied in Fig. 4a, observing that as $p_{T}$ is increased our system's output converges to the $p_{T}=\infty$ behaviour (i.e. Fig. 2b).

As our system operates in a regime where concentrations of some system species (namely $z$ and $z^{*}$ ) are low, the stochasticity of individual biochemical reactions may substantially influence its output. This phenomenon is frequently described as Intrinsic Noise (33), as it represents variability introduced within a given system. In Fig. 4b-e we perform tau-leaping Gillespie simulations of the system, setting $p_{T}=50$ cell $^{-1}$ and expressing each state variable in terms of integer copies per cell (rather than concentration). This follows from an average $E$. coli cell volume of $0.6 \mu \mathrm{m}^{3}$ meaning that a $1 \mathrm{nM}$ concentration corresponds to approximately 1 molecule per cell. We observe substantial variability in $z$ and $z^{*}$ concentrations (Fig. 4c), which drives variability in $I$ and $X$ (Fig. 4d), and through that $\hat{p}$ (Fig. $4 \mathrm{~b}$ ) and ultimately $G$ (Fig. 4e). In Fig. 4e we observe that stochastic treatment of this system increases its mean output level (from $G_{0}$ to $\hat{G}_{0}$ ), and (as an average of many simulations, $\bar{G})$ degrades the accuracy of adaptation to changing $\Delta$. This changed behaviour arises due to the timeaveraged value of $K z z^{*}$ in $\mathrm{Eq}(1 \mathrm{~b}, \mathrm{c})$ being reduced in the stochastic case. This intuitively follows from the fact that $\mathbb{E}\left[z z^{*}\right]=\mathbb{E}[z] \mathbb{E}\left[z^{*}\right]+\operatorname{cov}\left(z, z^{*}\right)$ (where $\mathbb{E}$ and cov are the expectation and covariance of a random variable respectively), and their covariance will be negative (due to negative inter-dependence introduced by $-K z z^{*}$ ), thus reducing the expected magnitude of the product $z z^{*}$ (for further analysis of this situation see
$(7,34))$.

\section{Discussion}

Our results demonstrate that the proposed switching control architecture is able to effectively reject a wide range disturbances to either translation or transcription rate. Similar switching controllers are employed in traditional engineering situations when a controlled variable needs to be maintained close to a set-point, and minimisation of energy used for control actuation is also important (30). For example, refrigeration/heating systems are often implemented by defining temperature limits above/below a set-point, outside of which the corresponding machinery is activated (35). Such implementations can be highly efficient, since minimal energy is consumed when the temperature is in the slack region near to its set-point (35). Analogously, for the biological controller outlined herein the two DNA flipping operations represent cellular machinery which is activated when the system leaves its slack region, and the consumption of energy (this time in the form of cellular resources) is minimal within the slack region.

While $\Delta_{p}, \Theta_{p}$ represent minimum disturbance limits that result in control saturation, the other limits described are solely constraints on the low-burden slack region in which the controller is designed to operate. These can be exceeded without degrading control capability, but come at the expense of increased protein production (and hence burden) in mode $m_{0}$. Tuning of system parameters would allow the system's set-point 
to be placed closer to the middle of this range. As $\Delta, \Theta$ grow the primary limit on the system's adaptative ability will thus be that imposed by the discretisation of DNA register state: $p$ cannot be made arbitrarily small, and so large $\Delta, \Theta$ cause a stepped response (Fig. 4a), particularly when the total number of DNA registers $\left(p_{T}\right)$ is small. Furthermore, regulation of $z$ by measurement protein species $D$ may lose linearity (and saturate) for large $\Delta, \Theta$, providing an additional upper limit on disturbance size. The system's adaptive capability was degraded when constituent reactions were simulated stochastically; this phenomenon has been demonstrated to depend upon a system's operating parameter regime (34), and thus would be a target for experimental parameter tuning. Our system's design is intended to minimise the burden it imposes on a host cell by operating in a state where $I, X$ (and potentially $D$ ) protein concentrations are small. However, in doing so trade-offs are being made in terms of consumption of different cellular resources. For example, maintaining a large number of DNA registers $\left(p_{T}\right)$ within a cell may also impact growth and other cellular processes.

One challenge posed by experimental implementation of the proposed control system is the creation of mRNA species $z$ and $z^{*}$ which encode genes and also bind to one another. Similar behaviours have been observed in some natural systems (36), though are at present beyond the capabilities of commonly used RNA engineering tools. In Supplementary Section 3 we analyse an analogous architecture in which each mRNA is co-expressed with a repressing sRNA (and thus $z$ and $z^{*}$ do not need to mutually bind). The design of each mRNA is thereby simplified, though tuning of sRNA binding strength (demonstrated in a number of recent synthetic biological studies $(37,26,27))$ is required instead.

Future work will include further stochastic analysis of the proposed control architecture's behaviour, as well as investigation of its parameter sensitivity. Experimental implementation would then likely require tuning of system parameters that are identified as being major determinants of performance, for example via Ribosome Binding Site variation or inclusion of protein degradation tags (i.e. to vary $\delta_{2}$ ) as was done in past implementations of Integrase/Excisionase controllers (24). In the broader sense, control mechanisms that utilise Integrase/Excisionase mediated DNA flipping may present interesting opportunities for low-burden (though some plasmid replication is required) dynamic information storage: Whilst information stored in the concentration of a protein (or phosphorylation state of a substrate) is diluted over time, the mean state of a DNA sequence is maintained (since it is copied in its present form). Future work may thus include development of different control schemes based upon the fundamental philosophy described herein, eventually leading to low-burden adaptive controllers with a diversity of synthetic biological applicatons (3).

\section{Supporting Information}

Supplementary Section 1: Parameter values used in simulations; Supplementary Section 2: Analysis of steadystate response to variation in $\Delta$ or $\Theta$; Supplementary Section 3: Approximating mRNA-mRNA annihilation junctions using sRNA.

\section{Acknowledgements}

The authors would like to thank Yili Qian, Theodore Grunberg, and Christian Cuba Samaniego, for discussion and advice relating to the ideas presented herein.

\section{Author Contributions}

H.S. concieved the idea and performed simulations. H.S. and A.P. wrote the manuscript.

\section{Funding}

H. Steel is supported by the General Sir John Monash Foundation. A. Papachristodoulou is supported by EPSRC project EP/M002454/1.

\section{References}

[1] D. Del Vecchio, A. J. Dy, and Y. Qian, "Control theory meets synthetic biology." J. R. Soc., Interface, vol. 13, no. 120, pp. 3-43, 2016.

[2] H. Steel, G. Lillacci, M. Khammash, and A. Papachristodoulou, "Challenges at the Interface of Control Engineering and Synthetic Biology," Proc. IEEE Conf. Decision Control, no. 1014-1023, 2017.

[3] M. Khammash, "An engineering viewpoint on biological robustness." BMC biology, vol. 14, no. 1, p. 22,2016 .

[4] T. M. Yi, Y. Huang, M. I. Simon, and J. Doyle, "Robust perfect adaptation in bacterial chemotaxis through integral feedback control." Proc. Natl. Acad. Sci. U., vol. 97, no. 9, pp. 4649-53, 2000.

[5] H. El-Samad, J. P. Goff, and M. Khammash, "Calcium homeostasis and parturient hypocalcemia: An integral feedback perspective," J. Theor. Biol., vol. 214, no. 1, pp. 17-29, 2002.

[6] H. Steel and A. Papachristodoulou, "Design Constraints for Biological Systems that Achieve Adaptation and Disturbance Rejection," IEEE Trans. Control Network Sys., vol. 5, no. 2, pp. 807-817, 2018.

[7] C. Briat, A. Gupta, and M. Khammash, "Antithetic Integral Feedback Ensures Robust Perfect Adaptation in Noisy Biomolecular Networks," Cell Systems, vol. 2, no. 1, pp. 15-26, 2016. 
[8] S. K. Aoki, G. Lillacci, A. Gupta, A. Baumschlager, and M. Khammash, "A universal rationally-designed biomolecular integral feedback controller for robust perfect adaptation," Nature, vol. 570, 2019.

[9] V. Hsiao, E. L. C. De Los Santos, W. R. Whitaker, J. E. Dueber, and R. M. Murray, "Design and implementation of a biomolecular concentration tracker," ACS Synthetic Biology, vol. 4, no. 2, pp. 150-161, 2015.

[10] F. Annunziata, A. Matyjaszkiewicz, G. Fiore, C. S. Grierson, L. Marucci, M. Di Bernardo, and N. J. Savery, "An Orthogonal Multi-input Integration System to Control Gene Expression in Escherichia coli," ACS Synthetic Biology, vol. 6, no. 10, pp. 1816-1824, 2017.

[11] Y. Qian and D. Del Vecchio, "Realizing integral control' in living cells: how to overcome leaky integration due to dilution?" Journal of The Royal Society Interface, vol. 15, no. 139, p. 20170902, 2018.

[12] J. Ang and D. R. McMillen, "Physical constraints on biological integral control design for homeostasis and sensory adaptation," Biophysical Journal, vol. 104, no. 2, pp. 505-515, 2013.

[13] C. C. Samaniego and E. Franco, "An ultrasensitive motif for robust closed loop control of biomolecular systems," 2017 IEEE 56th Annual Conference on Decision and Control, CDC 2017, pp. 5334-5340, 2017.

[14] Y. Qian, H. H. Huang, J. I. Jiménez, and D. Del Vecchio, "Resource Competition Shapes the Response of Genetic Circuits," ACS Synthetic Biology, vol. 6, no. 7, pp. 1263-1272, 2017.

[15] A. P. Darlington, J. Kim, J. I. Jiménez, and D. G. Bates, "Dynamic allocation of orthogonal ribosomes facilitates uncoupling of co-expressed genes," Nature Communications, vol. 9, no. 1, 2018.

[16] Y. Dublanche, K. Michalodimitrakis, N. Kummerer, M. Foglierini, and L. Serrano, "Noise in transcription negative feedback loops: simulation and experimental analysis," Mol Syst Biol, vol. 2, p. $41,2006$.

[17] F. Ceroni, R. Algar, G. B. Stan, and T. Ellis, "Quantifying cellular capacity identifies gene expression designs with reduced burden," Nature Methods, vol. 12, no. 5, pp. 415-418, 2015.

[18] C. Liao, A. E. Blanchard, and T. Lu, "An integrative circuithost modelling framework for predicting synthetic gene network behaviours," Nature Microbiology, vol. 2, no. December, 2017.
[19] A. Nystrom, A. Papachristodoulou, and A. Angel, "A Dynamic Model of Resource Allocation in Response to the Presence of a Synthetic Construct," ACS Synthetic Biology, vol. 7, pp. 12011210, 2018.

[20] C. Mcbride and D. D. Vecchio, "Resource Sensor Design for Quantifying Resource Competition in Genetic Circuits," Proceedings of the 58th IEEE Conference on Decision and Control., 2018.

[21] J. Bonnet, P. Subsoontorn, and D. Endy, "Rewritable digital data storage in live cells via engineered control of recombination directionality," Proceedings of the National Academy of Sciences, vol. 109, no. 23, pp. 8884-8889, 2012.

[22] N. Roquet, A. P. Soleimany, A. C. Ferris, S. Aaronson, and T. K. Lu, "Synthetic recombinasebased state machines in living cells," Science, 2016.

[23] P. Siuti, J. Yazbek, and T. K. Lu, "Synthetic circuits integrating logic and memory in living cells." Nature biotechnology, vol. 31, no. 5, pp. 448-52, 2013.

[24] T. Folliard, H. Steel, T. P. Prescott, G. Wadhams, L. J. Rothschild, and A. Papachristodoulou, "A synthetic recombinase-based feedback loop results in robust expression," ACS Synthetic Biology, 2017.

[25] D. K. Agrawal, X. Tang, A. Westbrook, R. Marshall, C. S. Maxwell, J. Lucks, V. Noireaux, C. L. Beisel, M. J. Dunlop, and E. Franco, "Mathematical Modeling of RNA-Based Architectures for Closed Loop Control of Gene Expression," ACS Synthetic Biology, vol. 7, pp. 1219-1228, 2018.

[26] C. L. Kelly, A. W. K. Harris, H. Steel, E. J. Hancock, J. T. Heap, and A. Papachristodoulou, "Synthetic negative feedback circuits using engineered small RNAs," Nucleic Acids Research, pp. 1-15, 2018.

[27] H.-H. Huang, Y. Qian, and D. D. Vecchio, "A quasi-integral controller for adaptation of genetic modules to variable ribosome demand," Nature Communications, vol. 9, no. 5415, 2018.

[28] R. D, McCardell, S. Huang, L. N. Green, and R. M. Murray, "Control of bacterial population density with population feedback and molecular sequestration," bioRxiv, p. 225045, 2017.

[29] D. Liberzon, Switching in Systems and Control. Springer, 2003.

[30] A. S. Morse, "Control using logic-based switching," in Trends in control. Springer, 1995, pp. 69-113. 
[31] J. E. Bowyer, V. Hsiao, W. W. Wong, and D. G. Bates, "Mechanistic modelling of a recombinasebased two-input temporal logic gate," Engineering Biology, vol. 1, pp. 40-50, 2017.

[32] J. E. Ferrell and S. H. Ha, "Ultrasensitivity part I: Michaelian responses and zero-order ultrasensitivity," Trends in Biochemical Sciences, vol. 39, no. 10, pp. 496-503, 2014.

[33] M. Elowitz, A. Levine, E. Siggia, and P. Swain, "Stochastic gene expression in a single cell," Science, vol. 297, no. 5584, pp. 1183-6, 2002.

[34] N. Olsman, F. Xiao, and J. C. Doyle, "Architectural Principles for Characterizing the Performance of Antithetic Integral Feedback Networks," iScience, vol. 14, pp. 277-291, 2019.

[35] M. A. Fayazbakhsh, F. Bagheri, and M. Bahrami, "Gray-box model for energy-efficient selection of set point hysteresis in heating, ventilation, air conditioning, and refrigeration controllers," Energy Conversion and Management, vol. 103, pp. 459$467,2015$.

[36] M. K. Thomason and G. Storz, "Bacterial Antisense RNAs: How Many Are There, and What Are They Doing?" Annual Review of Genetics, vol. 44, no. 1, pp. 167-188, 2010.

[37] D. Na, S. M. Yoo, H. Chung, H. Park, J. H. Park, and S. Y. Lee, "Metabolic engineering of Escherichia coli using synthetic small regulatory RNAs." Nature biotechnology, vol. 31, no. 2, pp. 170-4, 2013. 


\section{Low-burden biological feedback controllers for near-perfect adaptation}

\section{Supplementary Material}

Harrison Steel and Antonis Papachristodoulou* 


\section{Supplementary Section 1 Parameter Values}

\begin{tabular}{c|c|c|c} 
Parameter & Value & Unit & Source \\
\hline$\alpha_{2}$ & 1 & $\mathrm{nM} \mathrm{s}^{-1}$ & 1,2 \\
$\alpha_{1}$ & $2 \times 10^{-3}$ & $\mathrm{~s}^{-1}$ & 1,2 \\
$\beta_{I 1}$ & $6 \times 10^{-4}$ & $\mathrm{~s}^{-1}$ & 1,2 \\
$\beta_{I 2}$ & $1.2 \times 10^{-3}$ & $\mathrm{~s}^{-1}$ & 1,2 \\
$\beta_{X}$ & $2.4 \times 10^{-3}$ & $\mathrm{~s}^{-1}$ & 1,2 \\
$\gamma_{1}$ & 1 & $\mathrm{nM} \mathrm{s}^{-1}$ & 1,2 \\
$\gamma_{2}$ & 1 & $\mathrm{nM} \mathrm{s}^{-1}$ & 1,2 \\
$K$ & $3 \times 10^{-2}$ & $\mathrm{nM}^{-1} \mathrm{~s}^{-1}$ & 1,3 \\
$K_{I}$ & 30 & $\mathrm{nM}$ & 4,5 \\
$K_{X}$ & 20 & $\mathrm{nM}^{-1}$ & 4,5 \\
$\delta_{1}$ & $4.1 \times 10^{-3}$ & $\mathrm{~s}^{-1}$ & 1,6 \\
$\delta_{2}$ & $7.8 \times 10^{-4}$ & $\mathrm{~s}^{-1}$ & 4,7 \\
$\delta_{3}$ & $3.9 \times 10^{-4}$ & $\mathrm{~s}^{-1}$ & 1,7 \\
$r_{\text {on }}$ & $8 \times 10^{-5}$ & $\mathrm{~s}^{-1}$ & 4,5 \\
$r_{\text {off }}$ & $2 \times 10^{-4}$ & $\mathrm{~s}^{-1}$ & 4,5
\end{tabular}

Table S1: Nominal parameter values used throughout the main text, motivated in the text below.

The protein degradation/dilution rate $\left(\delta_{3}\right)$ is set as $3.9 \times 10^{-4} \mathrm{~s}^{-1}$ to correspond to dilution during growth with a 30 minute doubling time, typical for E. coli. ${ }^{7}$ The degradation of the recombinase proteins was set to twice this value, which can be achieved by attaching a fast-degradation tag to these proteins (e.g. as in ${ }^{4}$ ). The mRNA degradation rate $\left(\delta_{1}\right)$ is set as $4.1 \times 10^{-3} \mathrm{~s}^{-1}$ to represent a typical mRNA half-life of $2.8 \mathrm{~min}$ for E. coli growing in exponential phase. ${ }^{6}$ Rates for translation/transcription (i.e. $\alpha_{i}$ 's, $\beta_{i}$ 's, and $\gamma_{i}$ 's) were set to typical values for mid-high expression in E. coli ${ }^{6-8}$ which balance the respective $\mathrm{mRNA} /$ protein degradation/dilution rates for $E$. coli such that: mRNA copy numbers are on the order of $\sim 10$ per cell ${ }^{2}$ the strongly expressed output GFP is on the order of $\sim 1000$ per cell (as expected for strongly expressed proteins ${ }^{2}$ ); and concentrations of recombinase proteins are on the order of $10-100$ (a typical level for low-mid expression proteins ${ }^{2}$ ) on account of their fast-degradation tags. The value of $K$ was set based on models of sRNA-mRNA interactions in the literature, ${ }^{3}$ and falls into the range considered by recent studies of similar architectures. ${ }^{9}$ Recombinase rate parameters $\left(K_{I}, K_{X}, r_{o n}, r_{o f f}\right)$ were selected based on models fit in past studies of these systems. ${ }^{4,5}$ 


\section{Supplementary Section 2 Analysis of steady-state behaviour}

In this section we analyse Eq 6 of the main text to investigate the parameter-dependence of our control system's disturbance ability (in both $\Delta$ and $\Theta$ ).

\section{Rejection of Disturbance to $\Delta$}

We compute partial derivatives of $\mathrm{Eq}(6 \mathrm{a})$ with respect to $\Delta$, setting $y=\Delta \beta_{X} \mu(z) / \delta_{2}$ to give:

$$
\begin{gathered}
\frac{\partial}{\partial \Delta} \frac{\alpha_{1} \gamma_{1} \Delta \Theta p}{\delta_{2}}=\frac{\partial}{\partial \Delta}\left(\delta_{1} z+K z \mu(z)\right) \\
\frac{\alpha_{1} \gamma_{1} \Theta p}{\delta_{2}}+\frac{\alpha_{1} \gamma_{1} \Theta \Delta}{\delta_{2}} \frac{\partial p}{\partial \Delta}=\frac{\partial z}{\partial \Delta}\left(\delta_{1}+\frac{k \mu(z) \delta_{1}}{\delta_{1}+K z}\right) \\
\frac{\alpha_{1} \gamma_{1} \Theta}{\delta_{2}}\left(p+\Delta \frac{\partial p}{\partial \Delta}\right)=\frac{\partial z}{\partial \Delta} C
\end{gathered}
$$

where we have collected the term on the right hand side into $C$. Then:

$$
\begin{gathered}
\frac{\alpha_{1} \gamma_{1} \Theta}{\delta_{2}}\left(p+\Delta \frac{\partial p}{\partial y} \frac{\partial y}{\partial \Delta}\right)=\frac{\partial z}{\partial \Delta} C \\
\frac{\alpha_{1} \gamma_{1} \Theta}{\delta_{2}}\left(p+\Delta \frac{\partial p}{\partial y}\left(\frac{\beta_{X} \mu(z)}{\delta_{2}}-\frac{\beta_{X} \mu(z)}{\delta_{2}} \frac{K \Delta}{\left(\delta_{1}+K z\right)} \frac{\partial z}{\partial \Delta}\right)\right)=\frac{\partial z}{\partial \Delta} C
\end{gathered}
$$

which when the factor of $\frac{\partial z}{\partial \Delta}$ is isolated gives:

$$
\frac{\partial z}{\partial \Delta}=\frac{\frac{\alpha_{1} \gamma_{1} \Theta}{\delta_{2}}\left(p+\Delta \frac{\partial p}{\partial y} \frac{\beta_{X} \mu(z)}{\delta_{2}}\right)}{C+\frac{\alpha_{1} \gamma_{1} \Theta}{\delta_{2}} \frac{\partial p}{\partial y} \frac{\beta_{X} \mu(z)}{\delta_{2}} \frac{K \Delta^{2}}{\left(\delta_{1}+K z\right)}} .
$$

\section{Rejection of Disturbance to $\Theta$}

We now follow a similar process to the above for parameter $\Theta$ :

$$
\begin{gathered}
\frac{\partial}{\partial \Theta} \frac{\alpha_{1} \gamma_{1} \Delta \Theta p}{\delta_{2}}=\frac{\partial}{\partial \Theta}\left(\delta_{1} z+K z \mu(z)\right) \\
\frac{\alpha_{1} \gamma_{1} \Delta p}{\delta_{2}}+\frac{\alpha_{1} \gamma_{1} \Theta \Delta}{\delta_{2}} \frac{\partial p}{\partial \Theta}=\frac{\partial z}{\partial \Theta} C \\
\frac{\alpha_{1} \gamma_{1} \Delta}{\delta_{2}}\left(p+\Theta \frac{\partial p}{\partial y} \frac{\partial y}{\partial \Theta}\right)=\frac{\partial z}{\partial \Theta} C \\
\frac{\alpha_{1} \gamma_{1} \Delta}{\delta_{2}}\left(p+\Theta \frac{\partial p}{\partial y}\left(-\frac{\beta_{X} \mu(z)}{\delta_{2}} \frac{K \Delta}{\left(\delta_{1}+K z\right)} \frac{\partial z}{\partial \Theta}\right)\right)=\frac{\partial z}{\partial \Theta} C
\end{gathered}
$$

which when the factor of $\frac{\partial z}{\partial \Theta}$ is isolated gives:

$$
\frac{\partial z}{\partial \Theta}=\frac{\frac{\alpha_{1} \gamma_{1} \Delta}{\delta_{2}}(p)}{C+\frac{\alpha_{1} \gamma_{1} \Theta^{2}}{\delta_{2}} \frac{\partial p}{\partial y} \frac{\beta_{X} \mu(z)}{\delta_{2}} \frac{K \Delta}{\left(\delta_{1}+K z\right)}} .
$$

Comparing Rejection of $\Delta$ and $\Theta$

Since all parameters are positive and $\frac{\partial p}{\partial y}>0$ ( $p$ is an increasing function of $y$ ) we observe from Eq (S6) and Eq (S11) that when $\Delta, \Theta \approx 1$ have $\frac{\partial z}{\partial \Delta}>\frac{\partial z}{\partial \Theta}$. It therefore follows from the above that $\frac{\partial(\Delta \Theta p)}{\partial \Delta}>\frac{\partial(\Delta \Theta p)}{\partial \Theta}$, which means the system's steady-state output will be more sensitive to changes in $\Delta$ than $\Theta$ (as found in Fig. 2b,c of the main text). 


\section{Supplementary Section 3 Approximating mRNA-mRNA Annihila- tion}

A structural motif which we employ in this study is an mRNA-mRNA annihilation reaction (Fig. S1a), in which two mRNAs irreversibly bind to each other to prevent translation of either strand. Similar behaviours have been observed in some natural systems, ${ }^{10}$ though they have not yet been developed in a synthetic biological context. To overcome this experimental challenge here we briefly describe an equivalent RNA architecture (Fig. S1b) which additionally employs two sRNAs (as have been widely used in synthetic biological constructs ${ }^{1,11}$ ). In this case we co-express an sRNA with each mRNA, which targets the opposing mRNA. We can model this architecture with a system of differential equations of the form:
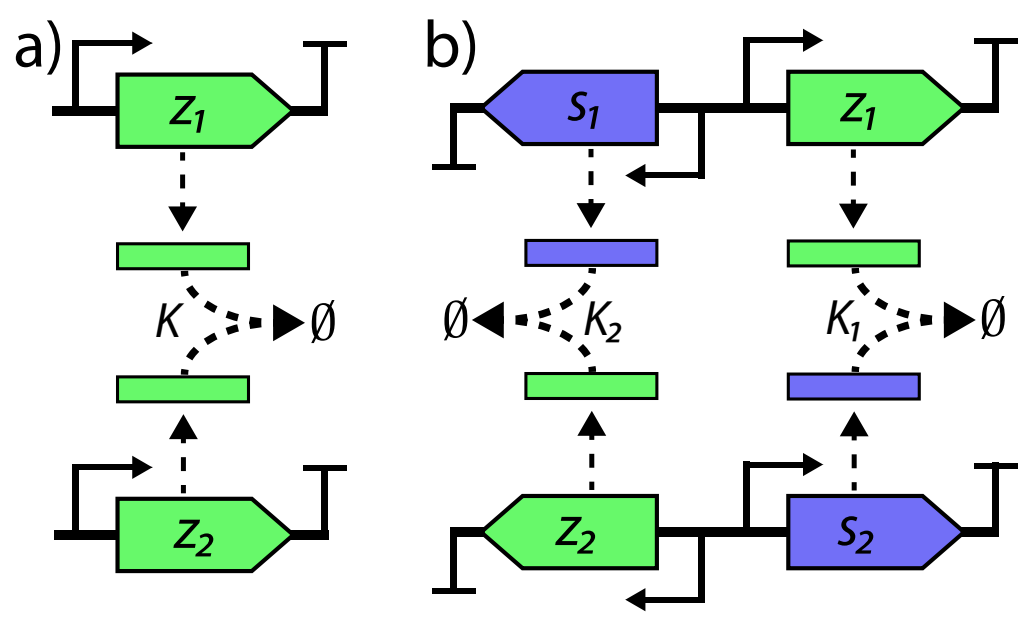

Figure S1: Circuit to approximate mRNA-mRNA Annihilation. a) An mRNA-mRNA annihilation interaction (paralleling that between $z$ and $z^{*}$ in the main text) for which we propose $\mathbf{b}$ ) an alternate architecture which employs sRNA to achieve equivalent functionality.

$$
\begin{aligned}
& \dot{z_{1}}=\alpha_{1}-\delta_{1 z} z_{1}-K_{1} z_{1} s_{2} \\
& \dot{z_{2}}=\alpha_{2}-\delta_{2 z} z_{2}-K_{2} z_{2} s_{1} \\
& \dot{s_{1}}=\alpha_{1}-\delta_{1 s} s_{1}-K_{2} z_{2} s_{1} \\
& \dot{s_{2}}=\alpha_{2}-\delta_{2 s} s_{2}-K_{1} z_{1} s_{2}
\end{aligned}
$$

where $\alpha_{i}$ are constitutive expression rates, $\delta_{i}$ are degradation rates, and $K_{i}$ are binding rates between each mRNA-sRNA pair. Examining Eq (S12) at steady state we find that $z_{1}$ must satisfy:

$$
0=\alpha_{1}-\delta_{1 z} z_{1}-\frac{K_{1} z_{1} \alpha_{2}}{\delta_{2 s}+K_{1} z_{1}}
$$

Meanwhile, the equivalent mRNA-mRNA version (Fig. S1a) of Eq (S12) would mirror Eq $(\mathrm{S} 12 \mathrm{a}, \mathrm{b})$ with $K_{1}=$ $K_{2}$, and $s_{1}$ and $s_{2}$ replaced by $z_{1}$ and $z_{2}$ respectively, that is:

$$
\begin{aligned}
& \dot{z_{1}}=\alpha_{1}-\delta_{1 z} z_{1}-K_{1} z_{1} z_{2} \\
& \dot{z_{2}}=\alpha_{2}-\delta_{2 z} z_{2}-K_{1} z_{2} z_{1}
\end{aligned}
$$

At steady state we therefore have:

$$
0=\alpha_{1}-\delta_{1 z} z_{1}-\frac{K_{1} z_{1} \alpha_{2}}{\delta_{2 z}+K_{1} z_{1}}
$$

which mirrors Eq (S13) up to the choice of $\delta_{2 z}$. We can therefore enforce equality between Eq (S13) and Eq (S15) by tuning the value of $K_{1}$ (and similarly, $K_{2}$ ) in the sRNA system (e.g. setting $K_{1}=\bar{K}_{1} \frac{\delta_{2 s}}{\delta_{2 z}}$ ).

If we have $K_{1}=\bar{K}_{1} \frac{\delta_{2 s}}{\delta_{2 z}}$ then Eq $(\mathrm{S} 12 \mathrm{a}, \mathrm{d})$ become:

$$
\begin{aligned}
& \dot{z_{1}}=\alpha_{1}-\delta_{1 z} z_{1}-\bar{K}_{1} \frac{\delta_{2 s}}{\delta_{2 z}} z_{1} s_{2} \\
& \dot{s_{2}}=\alpha_{2}-\delta_{2 s} s_{2}-\bar{K}_{1} \frac{\delta_{2 s}}{\delta_{2 z}} z_{1} s_{2}
\end{aligned}
$$


which, by setting $s_{2}=\overline{s_{2}} \frac{\delta_{2 z}}{\delta_{2} s}$ is transformed into:

$$
\begin{aligned}
& \dot{z_{1}}=\alpha_{1}-\delta_{1 z} z_{1}-\overline{K_{1}} z_{1} \overline{s_{2}} \\
& \dot{\overline{s_{2}}}=\frac{\delta_{2 s}}{\delta_{2 z}}\left(\alpha_{2}-\delta_{2 z} \overline{s_{2}}-\overline{K_{1}} z_{1} \overline{s_{2}}\right)
\end{aligned}
$$

which illustrates that the dynamics of the sRNA component of this system are scaled by a factor of $\frac{\delta_{2 s}}{\delta_{2 z}}$ when compared to the mRNA-mRNA system (i.e. Eq (S14)). The value of this scaling factor will depend on the particular sequences (and hence degradation rates) chosen for each sRNA, which will be influenced by the type of repressing sRNA chosen (e.g. sRNA that interact with Hfq chaperone proteins can be stabilised, leading to a reduced effective degradation rate $\left.{ }^{1,12}\right)$.

\section{References}

[1] C. L. Kelly, A. W. K. Harris, H. Steel, E. J. Hancock, J. T. Heap, and A. Papachristodoulou, "Synthetic negative feedback circuits using engineered small RNAs," Nucleic Acids Research, pp. 1-15, 2018.

[2] Y. Taniguchi, P. J. Choi, G.-w. Li, H. Chen, M. Babu, J. Hearn, A. Emili, and X. S. Xie, "Quantifying E. coli proteome and transcriptome with single-molecule sensitivity in single cells," Science, vol. 329, no. 5991, pp. 533-538, 2010.

[3] D. K. Agrawal, X. Tang, A. Westbrook, R. Marshall, C. S. Maxwell, J. Lucks, V. Noireaux, C. L. Beisel, M. J. Dunlop, and E. Franco, "Mathematical Modeling of RNA-Based Architectures for Closed Loop Control of Gene Expression," ACS Synthetic Biology, vol. 7, pp. 1219-1228, 2018.

[4] T. Folliard, H. Steel, T. P. Prescott, G. Wadhams, L. J. Rothschild, and A. Papachristodoulou, "A synthetic recombinase-based feedback loop results in robust expression," ACS Synthetic Biology, 2017.

[5] J. E. Bowyer, V. Hsiao, W. W. Wong, and D. G. Bates, "Mechanistic modelling of a recombinase-based two-input temporal logic gate," IET Engineering Biology, vol. 1, pp. 40-50, 2017.

[6] H. Chen, K. Shiroguchi, H. Ge, and X. S. Xie, "Genome-wide study of mRNA degradation and transcript elongation in Escherichia coli," Molecular Systems Biology, vol. 11, no. 1, pp. 781-781, 2015.

[7] S. T. Liang, M. Bipatnath, Y. C. Xu, S. L. Chen, P. Dennis, M. Ehrenberg, and H. Bremer, "Activities of constitutive promoters in Escherichia coli," Journal of Molecular Biology, vol. 292, no. 1, pp. 19-37, 1999.

[8] C. A. McCullen, J. N. Benhammou, N. Majdalani, and S. Gottesman, "Mechanism of positive regulation by DsrA and RprA small noncoding RNAs: Pairing increases translation and protects rpoS mRNA from degradation," Journal of Bacteriology, vol. 192, no. 21, pp. 5559-5571, 2010.

[9] N. Olsman, F. Xiao, and J. C. Doyle, "Architectural Principles for Characterizing the Performance of Antithetic Integral Feedback Networks," iScience, vol. 14, pp. 277-291, 2019.

[10] M. K. Thomason and G. Storz, "Bacterial Antisense RNAs: How Many Are There, and What Are They Doing?" Annual Review of Genetics, vol. 44, no. 1, pp. 167-188, 2010.

[11] H.-H. Huang, Y. Qian, and D. D. Vecchio, "A quasi-integral controller for adaptation of genetic modules to variable ribosome demand," Nature Communications, vol. 9, no. 5415, 2018.

[12] T. Soper, P. Mandin, N. Majdalani, S. Gottesman, and S. a. Woodson, "Positive regulation by small RNAs and the role of Hfq," Pnas, vol. 107, no. 21, pp. 2-7, 2010. 